Erdal Karapınar* and Andreea Fulga

\title{
An admissible Hybrid contraction with an Ulam type stability
}

https://doi.org/10.1515/dema-2019-0037

Received April 30, 2019; accepted September 12, 2019

Abstract: In this manuscript, we introduce a new hybrid contraction that unify several nonlinear and linear contractions in the set-up of a complete metric space. We present an example to indicate the genuine of the proved result. In addition, we consider Ulam type stability and well-posedness for this new hybrid contraction.

Keywords: admissible mappings, hybrid contractions, fixed point, metric space

MSC: 47H10, 54H25, 46J10

\section{Introduction and preliminaries}

In the last three-four decades, there is a blown out in the number of publications in metric fixed point theory. This fact forces researchers to find a way to combine, unify and merge the existing results in a proper way. In this paper, we aim to give an interesting example for this trend. We introduce a new hybrid contraction which not only combine and unify the several existing linear and nonlinear contractions but also extend these results.

Let $\Psi$ be the set of functions $\psi:[0, \infty) \rightarrow[0, \infty)$ such that

$\left(\Psi_{1}\right) \psi$ is non-decreasing;

$\left(\Psi_{2}\right)$ there are $i_{0} \in \mathbb{N}$ and $\delta \in(0,1)$ and a convergent series $\sum_{i=1}^{\infty} v_{i}$ such that $v_{i} \geq 0$ and

$$
\psi^{i+1}(t) \leq \delta \psi^{i}(t)+v_{i},
$$

for $i \geq i_{0}$ and $t \geq 0$.

Each $\psi \in \Psi$ is called a (c)-comparison function (see [1, 2]).

Lemma 1.1. [1] If $\psi \in \Psi$, then

(i) $\left(\psi^{n}(t)\right)_{n \in \mathbb{N}}$ converges to 0 as $n \rightarrow \infty$ for $t \geq 0$;

(ii) $\psi(t)<t$, for any $t \in \mathbb{R}^{+}$;

(iii) $\psi$ is continuous at 0 ;

(iv) the series $\sum_{k=1}^{\infty} \psi^{k}(t)$ is convergent for $t \geq 0$.

Let $\alpha: X \times X \rightarrow[0, \infty)$ be a function. We say that a mapping $f: X \rightarrow X$ is $\alpha$-orbital admissible ([3]) if

$$
\alpha(\chi, f \chi) \geq 1 \Rightarrow \alpha\left(f \chi, f^{2} \chi\right) \geq 1, \forall \chi \in \chi .
$$

\footnotetext{
`Corresponding Author: Erdal Karapınar: Department of Medical Research, China Medical University Hospital, 40402, Taichung, Taiwan; E-mail: erdalkarapinar@yahoo.com

Andreea Fulga: Department of Mathematics and Computer Sciences, Universitatea Transilvania Brasov, 500091, Brasov, Romania; E-mail: afulga@unitbv.ro
} 
An $\alpha$-orbital admissible mapping $f$ is called triangular $\alpha$-orbital admissible ([3]) if

$$
\alpha(x, y) \geq 1 \text { and } \alpha(y, f y) \geq 1 \Rightarrow \alpha(x, y) \geq 1,
$$

for every $x, y \in X$.

Lemma 1.2. Suppose that for a triangular $\alpha$-orbital admissible mapping $f: x \rightarrow x$ there exists $x_{0} \in x$ such that $\alpha\left(\chi_{0}, f \chi_{0}\right) \geq 1$. Then

$$
\alpha\left(x_{n}, x_{m}\right) \geq 1, \quad \text { for all } n, m \in \mathbb{N},
$$

where the sequence $\left\{\chi_{n}\right\}$ is defined by $\chi_{n+1}=f \chi_{n}, n \in \mathbb{N}$.

Definition 1.3. Let $\alpha: x \times X \rightarrow[0, \infty)$ be a mapping. The set $x$ is called regular with respect to $\alpha$ if for a sequence $\left\{x_{n}\right\}$ in $x$ such that $\alpha\left(x_{n}, x_{n+1}\right) \geq 1$, for all $n$ and $x_{n} \rightarrow x \in X$ as $n \rightarrow \infty$ we have $\alpha\left(x_{n}, x\right) \geq 1$ for all $n$.

\section{Main results}

We start with a definition of a new notion, namely "admissible hybrid contraction":

Definition 2.1. Let $(x, d)$ be a metric space. A self-mapping $f$ is called an admissible hybrid contraction, if there exist $\psi \in \Psi$ and $\alpha: X \times X \rightarrow[0, \infty)$ such that

$$
\alpha(x, y) d(f x, f y) \leq \psi\left(\mathcal{R}_{f}^{q}(x, y)\right),
$$

where $q \geq 0$ and $\lambda_{i} \geq 0, i=1,2,3,4,5$ such that $\sum_{i=1}^{5} \lambda_{i}=1$ and

$$
\mathcal{R}_{f}^{q} d(x, y)=\left\{\begin{array}{c}
{\left[\lambda_{1} d^{q}(x, y)(x, y)+\lambda_{2} d^{q}(x, f x)+\lambda_{3} d^{q}(y, f y)+\lambda_{4}\left(\frac{d(y, f y)(1+d(x, f x))}{1+d(x, y)}\right)^{q}+\lambda_{5}\left(\frac{d(y, f x)(1+d(x, f y))}{1+d(x, y)}\right)^{q}\right]^{\frac{1}{q}},} \\
\quad \text { or } q>0, x, y \in x \\
{[d(x, y)]^{\lambda_{1}} \cdot[d(x, f x)]^{\lambda_{2}} \cdot[d(y, f y)]^{\lambda_{3}} \cdot\left[\frac{d(y, f y)(1+d(x, f x))}{1+d(x, y)}\right]^{\lambda_{4}} \cdot\left[\frac{d(x, f y)+d(y, f x)}{2}\right]^{\lambda_{5}},} \\
\text { for } q=0, x, y \in x \backslash \operatorname{Fix}_{f}(x)
\end{array}\right.
$$

$\left(\right.$ Here $\left.\operatorname{Fix}_{f}(x)=\{x \in X: f \chi=\chi\}.\right)$

The concept of "admissible hybrid contraction" is inspired from the notion of "interpolative contractions", see e.g. [4-9] The main results of this manuscript is the following theorem:

Theorem 2.2. Let $(x, d)$ be a complete metric space and let $f$ be an admissible hybrid contraction, Suppose also that:

(i) $f$ is triangular $\alpha$-orbital admissible;

(ii) there exists $\chi_{0} \in X$ such that $\alpha\left(\chi_{0}, f \chi_{0}\right) \geq 1$;

(iii) either, $f$ is continuous, or

(iv) $f^{2}$ is continuous and $\alpha(f \chi, \chi) \geq 1$ for any $\chi \in \operatorname{Fix}_{f^{2}}(x)$.

Then $f$ has a fixed point.

Proof. Starting from an arbitrary point $x_{0}$ in $x$ we recursively set-up the sequence $\left\{x_{n}\right\}$, as $x_{n}=f^{n} x_{0}$ for all $n \in \mathbb{N}$. Supposing that there exists some $m \in \mathbb{N}$ such that $f v_{m}=x_{m+1}=x_{m}$, we find that $x_{m}$ is a fixed point of $f$ and the proof is finished. So, we can presume from now on that $x_{n} \neq x_{n-1}$ for any $n \in \mathbb{N}$. Under the assumption (i), $f$ is admissible hybrid contraction, if we substituting in (5) $x$ by $x_{n-1}$ and $y$ by $x_{n}$ we get

$$
\alpha\left(x_{n-1}, x_{n}\right) d\left(f x_{n-1}, f x_{n}\right) \leq \psi\left(\mathcal{R}_{f}^{q}\left(x_{n-1}, x_{n}\right)\right) .
$$


Taking into account that $f$ is triangular $\alpha$-orbital admissible, together with (4) holds and the above inequality becomes

$$
d\left(x_{n}, x_{n+1}\right) \leq \alpha\left(x_{n-1}, x_{n}\right) d\left(f x_{n-1}, f x_{n}\right)<\psi\left(\mathcal{R}_{f}^{q}\left(x_{n-1}, x_{n}\right) .\right.
$$

Case 1. For the case $q>0$ we have

$$
\begin{aligned}
\mathcal{R}_{f}^{q}\left(x_{n-1}, x_{n}\right)= & {\left[\lambda_{1} d^{q}\left(x_{n-1}, x_{n}\right)+\lambda_{2} d^{q}\left(x_{n-1}, f x_{n-1}\right)+\lambda_{3} d^{q}\left(x_{n}, f x_{n}\right)+\lambda_{4}\left(\frac{d\left(x_{n}, f x_{n}\right)\left(1+d\left(x_{n-1}, f x_{n-1}\right)\right)}{1+d\left(x_{n-1}, x_{n}\right)}\right)^{q}\right.} \\
& \left.+\lambda_{5}\left(\frac{d\left(x_{n}, f x_{n-1}\right)\left(1+d\left(x_{n-1}, f x_{n}\right)\right)}{1+d\left(x_{n-1}, x_{n}\right)}\right)^{q}\right]^{\frac{1}{q}} \\
= & {\left[\lambda_{1} d^{q}\left(x_{n-1}, x_{n}\right)+\lambda_{2} d^{q}\left(x_{n-1}, x_{n}\right)+\lambda_{3} d^{q}\left(x_{n}, x_{n+1}\right)+\lambda_{4}\left(\frac{d\left(x_{n}, x_{n+1}\right)\left(1+d\left(x_{n-1}, x_{n}\right)\right)}{1+d\left(x_{n-1}, x_{n}\right)}\right)^{q}\right.} \\
& \left.+\lambda_{5}\left(\frac{d\left(x_{n}, x_{n}\right)\left(1+d\left(x_{n-1}, x_{n+1}\right)\right)}{1+d\left(x_{n-1}, x_{n}\right)}\right)^{q}\right]^{\frac{1}{q}} \\
= & {\left[\lambda_{1} d^{q}\left(x_{n-1}, x_{n}\right)+\lambda_{2} d^{q}\left(x_{n-1}, x_{n}\right)+\lambda_{3} d^{q}\left(x_{n}, x_{n+1}\right)+\lambda_{4}\left(d\left(x_{n}, x_{n+1}\right)\right)^{q}\right]^{\frac{1}{q}} } \\
= & {\left[\left(\lambda_{1}+\lambda_{2}\right) d^{q}\left(x_{n-1}, x_{n}\right)+\left(\lambda_{3}+\lambda_{4}\right) d^{q}\left(x_{n}, x_{n+1}\right)\right]^{1 / q}, }
\end{aligned}
$$

and from (8) we get

$$
\begin{aligned}
d\left(x_{n}, x_{n+1}\right) & \leq \alpha\left(x_{n-1}, x_{n}\right) d\left(f x_{n-1}, f x_{n}\right) \\
& <\psi\left(\mathcal{R}_{f}^{q}\left(x_{n-1}, x_{n}\right)\right) \\
& =\psi\left(\left[\left(\lambda_{1}+\lambda_{2}\right) d^{q}\left(x_{n-1}, x_{n}\right)+\left(\lambda_{3}+\lambda_{4}\right) d^{q}\left(x_{n}, x_{n+1}\right)\right]^{1 / q}\right) .
\end{aligned}
$$

If we suppose that $d\left(x_{n-1}, x_{n}\right) \leq d\left(x_{n}, x_{n-1}\right)$, since $\psi$ is a nondecreasing function,

$$
\begin{aligned}
d\left(x_{n}, x_{n+1}\right) & \leq \alpha\left(x_{n-1}, x_{n}\right) d\left(f x_{n-1}, f x_{n}\right) \\
& \leq \psi\left(\left[\left(\lambda_{1}+\lambda_{2}\right) d^{q}\left(x_{n-1}, x_{n}\right)+\left(\lambda_{3}+\lambda_{4}\right) d^{q}\left(x_{n}, x_{n+1}\right)\right]^{1 / q}\right) \\
& \left.\leq \psi\left(\left[\lambda_{1}+\lambda_{2}+\lambda_{3}+\lambda_{4}\right) d^{q}\left(x_{n}, x_{n+1}\right)\right]^{1 / q}\right) \\
& =\psi\left(\left(\lambda_{1}+\lambda_{2}+\lambda_{3}+\lambda_{4}\right)^{1 / q} d\left(x_{n}, x_{n+1}\right)\right) \\
& <\left(\lambda_{1}+\lambda_{2}+\lambda_{3}+\lambda_{4}\right)^{1 / q} d\left(x_{n}, x_{n+1}\right) \\
& \leq d\left(x_{n}, x_{n+1}\right)
\end{aligned}
$$

which is a contradiction. Therefore, for every $n \in \mathbb{N}$ we have

$$
d\left(x_{n}, x_{n+1}\right)<d\left(x_{n-1}, x_{n}\right),
$$

and the inequality (8) yields

$$
\begin{aligned}
d\left(x_{n}, x_{n+1}\right) & \leq \psi\left(\left[\left(\lambda_{1}+\lambda_{2}\right) d^{q}\left(x_{n-1}, x_{n}\right)+\left(\lambda_{3}+\lambda_{4}\right) d^{q}\left(x_{n}, x_{n+1}\right)\right]^{1 / q}\right) \\
& \left.<\psi\left(\left[\lambda_{1}+\lambda_{2}+\lambda_{3}+\lambda_{4}\right) d^{q}\left(x_{n-1}, x_{n}\right)\right]^{1 / q}\right) \\
& \leq \psi\left(\left(\lambda_{1}+\lambda_{2}+\lambda_{3}+\lambda_{4}\right)^{1 / q} d\left(x_{n-1}, x_{n}\right)\right) \\
& \leq \psi\left(d\left(x_{n-1}, x_{n}\right)\right)<\psi^{2}\left(d\left(x_{n-2}, x_{n-1}\right)\right) \\
& \cdots \\
& <\psi^{n}\left(d\left(x_{0}, x_{1}\right)\right) .
\end{aligned}
$$

Let now, $m, p \in \mathbb{N}$ such that $p>m$. By the triangle inequality and since $d\left(x_{m}, x_{m+1}\right)<\psi^{m}\left(d\left(x_{0}, x_{1}\right)\right)$ for any $m \in \mathbb{N}$, we have

$$
\begin{aligned}
d\left(x_{m}, x_{p}\right) & \leq d\left(x_{m}, x_{m+1}\right)+d\left(x_{m+1}, x_{m+2}\right)+\ldots+d\left(x_{p-1}, x_{p}\right) \\
& =\sum_{j=m}^{p-1} d\left(x_{j}, x_{j+1}\right) \leq \sum_{j=m}^{p-1} \psi^{j}\left(d\left(x_{0}, x_{1}\right)\right) .
\end{aligned}
$$

Since $\psi$ is a $c$-comparison function the series $\sum_{j=0}^{\infty} \psi^{j}\left(d\left(x_{0}, x_{1}\right)\right)$ is convergent, so that, denoting by $\mathcal{S}_{n}=$ $\sum_{j=0}^{n} \psi^{j}\left(d\left(x_{0}, x_{1}\right)\right)$ the above inequality becomes:

$$
d\left(x_{m}, x_{p}\right) \leq \mathcal{S}_{p-1}-\mathcal{S}_{m-1},
$$


and as $m, p \rightarrow \infty$ we get

$$
d\left(x_{m}, x_{p}\right) \rightarrow 0
$$

which tells us that $\left\{x_{n}\right\}$ is a Cauchy sequence on a complete metric space, so that, there exists $z$ such that

$$
\lim _{n \rightarrow \infty} d\left(x_{n}, z\right)=0
$$

We will prove that this point $z$ is a fixed point of $f$. If $f$ is continuous, (due to assumption (iii))

$$
\lim _{n \rightarrow \infty} d\left(x_{n+1}, f z\right)=\lim _{n \rightarrow \infty} d\left(x_{n}, f x_{n}\right)=0,
$$

so, we get that $f z=z$, that is, $z$ is a fixed point of $f$.

In the alternative hypothesis, that $f^{2}$ is continuous we have $f^{2} z=\lim _{n \rightarrow \infty} f^{2} x_{n}=z$ and we want to show that $f z=z$. Supposing that, on the contrary, $f z \neq z$, we have from (5)

$$
\begin{aligned}
d(z, f z) & =d\left(f^{2} z, f z\right) \leq \alpha(f z, z) d(f z, z) \\
\leq & \psi\left(\mathcal{R}_{f}^{q}(f z, z)\right)<\mathcal{R}_{f}^{q}(f z, z) \\
& =\left[\lambda_{1} d^{q}(f z, z)+\lambda_{2} d^{q}\left(f z, f^{2} z\right)+\lambda_{3} d^{q}(z, f z)+\lambda_{4}\left(\frac{d(z, f z)\left(1+d\left(f z, f^{2} z\right)\right)}{1+d(f z, z)}\right)^{q}+\lambda_{5}\left(\frac{d\left(z, f^{2} z\right)(1+d(f z, f z))}{1+d(f z, z)}\right)^{q}\right]^{\frac{1}{q}} \\
& =\left[\lambda_{1} d^{q}(f z, z)+\lambda_{2} d^{q}(f z, z)+\lambda_{3} d^{q}(z, f z)+\lambda_{4}\left(\frac{d(z, f z)(1+d(f z, z))}{1+d(f z, z)}\right)^{q}+\lambda_{5}\left(\frac{d(z, z)(1+d(f z, f z))}{1+d(f z, z)}\right)^{q}\right]^{\frac{1}{q}} \\
& =\left[\left(\lambda_{1}+\lambda_{2}+\lambda_{3}+\lambda_{4}\right) d^{q}(f z, z)\right]^{\frac{1}{q}} \\
& =\left[\left(\lambda_{1}+\lambda_{2}+\lambda_{3}+\lambda_{4}\right)\right]^{\frac{1}{q}} d(f z, z) \\
\leq & d(f z, z) .
\end{aligned}
$$

This is a contradiction, so that $f z=z$.

Case 2. For the case $q=0$ taking $x=x_{n-1}$ and $y=x_{n}$ we have

$$
\begin{aligned}
& \mathcal{R}_{f}^{q}\left(x_{n-1}, x_{n}\right)=\left[d\left(x_{n-1}, x_{n}\right)\right]^{\lambda_{1}} \cdot\left[d\left(x_{n-1}, f x_{n-1}\right)\right]^{\lambda_{2}} \cdot\left[d\left(x_{n}, f x_{n}\right)\right]^{\lambda_{3}} \cdot\left[\frac{d\left(x_{n}, f x_{n}\right)\left(1+d\left(x_{n-1}, f x_{n-1}\right)\right)}{1+d\left(x_{n-1}, x_{n}\right)}\right]^{\lambda_{4}} \cdot\left[\frac{\left.d\left(x_{n-1}, f x_{n}\right)+d\left(x_{n}, f x_{n-1}\right)\right)}{2}\right]^{\lambda_{5}} \\
& \leq\left[d\left(x_{n-1}, x_{n}\right)\right]^{\lambda_{1}} \cdot\left[d\left(x_{n-1}, x_{n}\right)\right]^{\lambda_{2}} \cdot\left[d\left(x_{n}, x_{n+1}\right)\right]^{\lambda_{3}} \cdot\left[\frac{d\left(x_{n}, x_{n+1}\right)\left(1+d\left(x_{n-1}, x_{n}\right)\right)}{1+d\left(x_{n-1}, x_{n}\right)}\right]^{\lambda_{4}} \cdot\left[\frac{\left.d\left(x_{n-1}, x_{n}\right)+d\left(x_{n}, x_{n+1}\right)+d\left(x_{n}, x_{n}\right)\right)}{2}\right]^{\lambda_{5}} \\
& \leq\left[d\left(x_{n-1}, x_{n}\right)\right]^{\lambda_{1}} \cdot\left[d\left(x_{n-1}, x_{n}\right)\right]^{\lambda_{2}} \cdot\left[d\left(x_{n}, x_{n+1}\right)\right]^{\lambda_{3}} \cdot\left[\frac{d\left(x_{n}, x_{n+1}\right)\left(1+d\left(x_{n-1}, x_{n}\right)\right)}{1+d\left(x_{n-1}, x_{n}\right)}\right]^{\lambda_{4}} \cdot \frac{\left[d\left(x_{n-1}, x_{n}\right)\right]^{\lambda_{5}}+\left[d\left(x_{n}, x_{n+1}\right)\right]^{\lambda_{5}}}{2} \\
& \leq\left[d\left(x_{n-1}, x_{n}\right)\right]^{\lambda_{1}+\lambda_{2}} \cdot\left[d\left(x_{n}, x_{n+1}\right)\right]^{\lambda_{3}+\lambda_{4}} \cdot \frac{\left[d\left(x_{n-1}, x_{n}\right)\right]^{\lambda_{5}}+\left[d\left(x_{n}, x_{n+1}\right)\right]^{\lambda_{5}}}{2}
\end{aligned}
$$

and from (5)

$$
d\left(x_{n}, x_{n+1}\right) \leq \alpha\left(x_{n-1}, x_{n}\right) d\left(f x_{n-1}, f x_{n}\right) \leq \psi\left(\mathcal{R}_{f}^{q}\left(x_{n-1}, x_{n}\right)\right) .
$$

As in the first case, we have that $d\left(x_{n-1}, x_{n}\right)>d\left(x_{n}, x_{n+1}\right)$ since in the contrary case we have a contradiction. Indeed, if we suppose ad absurdum that $d\left(x_{n-1}, x_{n}\right) \leq d\left(x_{n}, x_{n+1}\right)$, we have

$$
\left.\left.d\left(x_{n}, x_{n+1}\right)<\psi\left(\mathcal{R}_{f}^{q}\left(x_{n-1}, x_{n}\right)\right)<\left[d\left(x_{n}, x_{n+1}\right)\right)\right]^{\lambda_{1}+\lambda_{2} \lambda_{3}+\lambda_{4}+\lambda_{5}}=d\left(x_{n}, x_{n+1}\right)\right)
$$

which is a contradiction. Then from (14) we obtain

$$
d\left(x_{n}, x_{n+1}\right) \leq \psi\left(\mathcal{R}_{f}^{q}\left(x_{n-1}, x_{n}\right)\right)<\psi\left(d\left(x_{n-1}, x_{n}\right)\right)
$$

and inductively we get

$$
d\left(x_{n}, x_{n+1}\right) \leq \psi^{n}\left(d\left(x_{0}, x_{1}\right)\right) .
$$

By using the same arguments as the case $q>0$ we shall easily obtain that $\left\{x_{n}\right\}$ is a Cauchy sequence in a complete metric space and so, there exists $z$ such that $\lim _{n \rightarrow \infty} \chi_{n}=z$. 
We claim that $z$ is a fixed point of $f$.

Under the assumption that $f$ is continuous we have

$$
\lim _{n \rightarrow \infty} d\left(x_{n+1}, f z\right)=\lim _{n \rightarrow \infty} d\left(f x_{n}, f z\right)=0,
$$

and together with the uniqueness of limit, $f z=z$. Also, if $f^{2}$ is continuous, as in case (1) we have that $f z=z$ and then

$$
\begin{aligned}
d(z, f z) & =d\left(f^{2} z, f z\right) \leq \alpha(f z, z) d\left(f^{2} z, f z\right) \leq \psi\left(\mathcal{R}_{f}^{q}\left(f^{2} z, f z\right)\right) \\
& \leq \psi\left([d(z, f z)]^{\lambda_{1}+\lambda_{2}+\lambda_{3}+\lambda_{4}+\lambda_{5}}\right)<d(z, f z) .
\end{aligned}
$$

This contradiction shows us that $z=f z$.

Example. Let $x=[0,2], d: x \times x \rightarrow[0, \infty)$ be the usual metric, $d(x, y)=|x-y|$ for all $x, y \in x$ and the mapping $f: x \rightarrow x$ be defined by $f(x)=\left\{\begin{array}{ll}2 / 3, & \text { if } x \in[0,1] \\ x / 2, & \text { if } x \in(1,2]\end{array}\right.$. Consider also a function $\alpha(x, y)=$ $\left\{\begin{array}{ll}2, & \text { if } x, y \in[0,1] \\ 1, & \text { if } x=0, y=2 \text { and the comparison function } \psi:[0, \infty) \rightarrow[0, \infty), \psi(t)=t / 5 . \text { We can easily observe } \\ 0, & \text { otherwise }\end{array}\right.$. that the assumptions (i) and (ii) are satisfied and since $f^{2}(x)=2 / 3$ is continuous, the assumption (iv) is also verified. For any $x, y \in[0,1]$ we have $d(f x, f y)=0$ so, the inequality (5) holds. For $\chi=0$ and $y=2$, we have

$$
\begin{aligned}
\alpha(0,2) d(f 0, f 2) & =\alpha(0,2) d(2 / 3,1)=\frac{1}{3}<\frac{1}{5} \sqrt{\frac{505}{81}}=\frac{1}{5} \sqrt{\frac{1}{4}\left(4+\frac{4}{9}+1+\frac{64}{81}\right)} \\
& =\frac{1}{5}\left[\frac{1}{4} d^{2}(0,2)+\frac{1}{4} d^{2}(0, f 0)++\frac{1}{4} d^{2}(2, f 2)+\frac{1}{4}\left(\frac{d(2, f 0)(1+d(0, f 2))}{1+d(0,2)}\right)^{2}\right]^{1 / 2} .
\end{aligned}
$$

In all other cases, $\alpha(x, y)=0$ and (5) is obviously satisfied. Thus, letting $\lambda_{1}=\lambda_{2}=\lambda_{3}=\lambda_{5}=\frac{1}{4}, \lambda_{4}=0$ and $q=2$ we obtain that $f$ is an admissible hybrid contraction which satisfies the assumptions (i), (ii), (iv) of Theorem 2.2 and then $\chi=0$ is the fixed point of $f$.

Theorem 2.3. Let $(x, d)$ be a complete metric space and let $f$ be an admissible hybrid contraction, Suppose also that:

1. $f$ is triangular $\alpha$-orbital admissible;

2. there exists $\chi_{0} \in X$ such that $\alpha\left(\chi_{0}, f \chi_{0}\right) \geq 1$;

3. $(x, d)$ is regular with respect to $\alpha$.

Then $f$ possesses a fixed point.

Proof. Following the lines in the proof of Theorem 2.2, we already know that for any $q \geq 0$, the sequence $\left\{x_{n}\right\}$ is Cauchy, and due to the completeness of the metric space $(x, d)$, there exists a point $z$ such that $\lim _{n \rightarrow \infty} d\left(x_{n}, z\right)=$ 0 . Since the space $x$ is regular with respect to $\alpha$, inequality (5) together with the triangular inequality gives us

$$
d(z, f z) \leq d\left(z, \chi_{n+1}\right)+d\left(\chi_{n+1}, f z\right) \leq \alpha\left(\chi_{n}, z\right) d\left(f \chi_{n}, f z\right) \leq \psi\left(\mathcal{R}_{f}^{q}\left(\chi_{n}, z\right)\right) \leq \mathcal{R}_{f}^{q}\left(\chi_{n}, z\right) .
$$

Again, we have to consider two separate cases. For the case $p>0$,

$$
\begin{aligned}
\mathcal{R}_{f}^{q}\left(\chi_{n}, z\right) & =\left[\lambda_{1} d^{q}\left(x_{n}, z\right)+\lambda_{2} d^{q}\left(\chi_{n}, f \chi_{n}\right)+\lambda_{3} d^{q}(z, f z)+\lambda_{4}\left(\frac{d(z, f z)\left(1+d\left(x_{n}, f_{x_{n}}\right)\right)}{1+d\left(\chi_{n}, z\right)}\right)^{q}+\lambda_{5}\left(\frac{d\left(z, f x_{n}\right)\left(1+d\left(x_{n}, f z\right)\right)}{1+d\left(x_{n}, z\right)}\right)^{q}\right]^{\frac{1}{q}} \\
& =\left[\lambda_{1} d^{q}\left(x_{n}, z\right)+\lambda_{2} d^{q}\left(x_{n}, x_{n+1}\right)+\lambda_{3} d^{q}(z, f z)+\lambda_{4}\left(\frac{d(z, f z)\left(1+d\left(x_{n}, x_{n+1}\right)\right)}{1+d\left(\chi_{n}, z\right)}\right)^{q}+\lambda_{5}\left(\frac{d\left(z, x_{n+1}\right)\left(1+d\left(x_{n}, f z\right)\right)}{1+d\left(x_{n}, z\right)}\right)^{q}\right]^{\frac{1}{q}} .
\end{aligned}
$$

Since $\lim _{n \rightarrow \infty} \mathcal{R}_{f}^{q}\left(\chi_{n}, z\right)=\left(\lambda_{3}+\lambda_{4}\right) d(z, f z)$, letting $n \rightarrow \infty$ in (16) we obtain $d(z, f z)<d(z, f z)$ which implies that $f z=z$.

Similarly, for the case $q=0$, we get $\lim _{n \rightarrow \infty} \mathcal{R}_{f}^{q}\left(\chi_{n}, z\right)=0$ and then $d(z, f z)=0$. 
Corollary 2.4. Let $(x, d)$ be a complete metric space and the functions $\psi \in \Psi$ and $\alpha: x \times x \rightarrow[0, \infty)$. Let $f$ be a self map on $x$ such that:

(i) $f$ is triangular $\alpha$-orbital admissible;

(ii) there exists $\chi_{0} \in X$ such that $\alpha\left(\chi_{0}, f \chi_{0}\right) \geq 1$;

(iii) either, $f$ is continuous, or

(iv) $f^{2}$ is continuous and $\alpha(f \chi, \chi) \geq 1$ for any $\chi \in$ Fix $_{f^{2}}(x)$.

If one of the below conditions $\left(c_{1}\right)-\left(c_{3}\right)$ is satisfied, then $f$ has a fixed point $z \in X$, that is, $f z=z$.

$\left(c_{1}\right) \alpha(x, y) d(x, y) \leq \psi\left(\mathcal{A}_{f}^{q}(x, y)\right)$, where $a_{1}, a_{2}, a_{3}, a_{4}$ are non-negative real such that $a_{1}+a_{2}+a_{3}+a_{4}=1$ and

$$
\mathcal{A}_{f}^{q} d(x, y)=\left\{\begin{array}{l}
{\left[a_{1} d^{q}(\chi, y)(x, y)+a_{2} d^{q}(\chi, f \chi)+a_{3} d^{q}(y, f y)+a_{4}\left(\frac{d(y, f y)(1+d(x, f x))}{1+d(x, y)}\right)^{q}\right]^{\frac{1}{q}}, \text { for } q>0, x, y \in X} \\
{[d(x, y)]^{a_{1}} \cdot[d(\chi, f x)]^{a_{2}} \cdot[d(y, f y)]^{a_{3}} \cdot\left[\frac{d(y, f y)(1+d(x, f x))}{1+d(x, y)}\right]^{a_{4}}, \text { for } q=0, x, y \in X \backslash \text { Fix }_{f}(x)}
\end{array}\right.
$$

$\left(c_{2}\right) \alpha(\chi, y) d(x, y) \leq \psi\left(\mathcal{B}_{f}^{q}(\chi, y)\right)$, where $b_{1}, b_{2}, b_{3}$ are non-negative real such that $b_{1}+b_{2}+b_{3}=1$ and

$$
\mathcal{B}_{f}^{q} d(x, y)=\left\{\begin{array}{l}
{\left[b_{1} d^{q}(x, y)(x, y)+b_{2} d^{q}(x, f \chi)+b_{3} d^{q}(y, f y)\right]^{\frac{1}{q}}, \quad \text { for } q>0, x, y \in X} \\
{[d(x, y)]^{b_{1}} \cdot[d(\chi, f x)]^{b_{2}} \cdot[d(y, f y)]^{b_{3}}, \quad \text { for } q=0, \chi, y \in X \backslash F_{i x}(x) .}
\end{array}\right.
$$

$\left(c_{3}\right) \alpha(\chi, y) d(\chi, y) \leq \psi\left(\mathcal{B}_{f}^{q}(\chi, y)\right)$, where $c_{1}, c_{2}$ are non-negative real numbers such that $c_{1}+c_{2}=1$ and

$$
\mathcal{C}_{f}^{q} d(x, y)=\left\{\begin{array}{l}
{\left[c_{1} d^{q}(x, f \chi)+c_{2} d^{q}(y, f y)\right]^{\frac{1}{q}}, \quad \text { for } q>0, x, y \in x} \\
{[d(x, f x)]^{c_{1}} \cdot[d(y, f y)]^{c_{2}}, \quad \text { for } q=0, x, y \in x \backslash \text { Fix }_{f}(x) .}
\end{array}\right.
$$

We can get a series of corollaries, considering in Corollary 2.4 by assigning $\psi \in \Psi$ properly, for example, by taking $\psi(t)=k t$ for any $t \geq 0$ with $k \in[0,1)$, and/or $\alpha(\chi, y)=1$ or both. Since it is apparent we skip the details.

Theorem 2.5. If in Theorems 2.2 and 2.3, in the case $q>0$, we assume supplementary that

$$
\alpha(x, y) \geq 1
$$

for any $x, y \in$ Fix $_{f}(x)$ then the fixed point of $f$ is unique.

Proof. Let $v \in X$ be another fixed point of $f$, different from $z$. By replacing in (5), and taking into account the additional hypotheses, we have

$$
\begin{aligned}
d(z, v) \leq \alpha(z, v)(f z, f v) \leq \psi\left(\mathcal{R}_{f}^{q}(z, v)\right)<\mathcal{R}_{f}^{q}(z, v)= & {\left[\lambda_{1} d^{q}(z, v) \lambda_{2} d^{q}(z, f z)+\lambda_{3} d^{q}(v, f v)+\right.} \\
& \left.+\lambda_{4}\left(\frac{d(v, f v)(1+d(z, f z))}{1+d(z, v)}\right)^{q}+\lambda_{5}\left(\frac{d(v, f z)(1+d(z, f v))}{1+d(z, v)}\right)^{q}\right]^{\frac{1}{q}} \\
= & {\left[\lambda_{1} d^{q}(z, v)+\lambda_{2} d^{q}(z, z)+\lambda_{3} d^{q}(v, v)+\right.} \\
& \left.+\lambda_{4}\left(\frac{d(v, v)(1+d(z, z))}{1+d(z, v)}\right)^{q}+\lambda_{5}\left(\frac{d(v, z)(1+d(z, v))}{1+d(z, v)}\right)^{q}\right]^{\frac{1}{q}} \\
= & d(z, v)\left(\lambda_{1}+\lambda_{5}\right)^{1 / q} \leq d(z, v),
\end{aligned}
$$

which is a contradiction. Thus, $z=v$, so that $f$ possesses exactly one fixed point.

Example. Let $x=\{a, 6, c, d\}$ and $d: x \times x \rightarrow[0, \infty)$ such that $d(x, y)=d(y, x), d(x, x)=0$ for any $x, y \in x$ and

$$
d(x, y)= \begin{cases}1, & \text { if }(x, y) \in\{(a, b),(b, c),(c, d)\} \\ 2, & \text { if }(x, y) \in\{(a, c),(b, d)\} \\ 3, & \text { if }(x, y) \in\{(a, d)\}\end{cases}
$$


On metric space $(x, d)$ let us define the self-mapping $f$ by $f(a)=f(6)=a, \quad f(c)=d, \quad f(d)=6$. Consider also a function $\alpha: X \times X \rightarrow[0, \infty)$, where $\alpha(\chi, a)=\alpha(a, \chi)=3$ for any $\chi \in X, \alpha(6, d)=1, \alpha(\chi, y)=0$ otherwise and the comparison function $\psi:[0, \infty) \rightarrow[0, \infty), \psi(t)=\sqrt[4]{\frac{3}{4}} t$. Since neither $f$, nor $f^{2}$ are continuous, Theorem 2.2 cannot be applied. On the other hand, is easy to see that $f$ is triangular $\alpha$-orbital admissible and also the assumptions (2), (3) from Theorem 2.3 are satisfied. Considering $q=0, \lambda_{1}=\lambda_{2}=\lambda_{3}=\lambda_{4}=1 / 4$ and $\lambda_{5}=0$ and taking into account the definition of function $\alpha$, we remark that the only interesting case is for $x=6$ and $y=d$. We have in this case:

$$
\begin{aligned}
\alpha(6, d) d(f 6, f d)=d(a, b) & =1<\sqrt{2}=\sqrt[4]{\frac{3}{4}} \cdot 2^{1 / 4} \cdot 1 \cdot 2^{1 / 4} \cdot\left(\frac{4}{3}\right)^{1 / 4} \\
& =\sqrt[4]{\frac{3}{4}}[d(6, d)]^{\lambda_{1}} \cdot[d(6, f 6)]^{\lambda_{2}} \cdot[d(d, f d)]^{\lambda_{3}} \cdot\left[\frac{d(d, f d)(1+d(6, f 6))}{1+d(6, d)}\right]^{\lambda_{4}} \\
& =\psi\left([d(6, d)]^{\lambda_{1}} \cdot[d(6, f 6)]^{\lambda_{2}} \cdot[d(d, f d)]^{\lambda_{3}} \cdot\left[\frac{d(d, f d)(1+d(b, f 6))}{1+d(6, d)}\right]^{\lambda_{4}}\right) .
\end{aligned}
$$

Consequently, the map $f$ has a fixed point, that is $\chi=a$.

\section{Ulam type stability}

Considered as a type of data dependence, the notion of Ulam stability was started by Ulam [10, 11] and deveped by Hyers [12], Rassias [13], etc In this section we investigate the general Ulam type stability in sense of a fixed point problem.

Suppose that $f: X \rightarrow X$ is a self-mapping on a metric space $(x, d)$. The fixed point problem

$$
x=f x,
$$

has the general Ulam type stability if and only if there exists an increasing function $\rho:[0, \infty) \leftrightarrow[0, \infty)$, continuous at 0 with $\rho(0)=0$ such that for every $\varepsilon>0$ and for each $y^{\star} \in X$ which satisfies the inequality

$$
d\left(y^{*}, f y^{*}\right) \leq \varepsilon
$$

there exists a solution $z \in X$ of (20) such that

$$
d\left(z, y^{\star}\right) \leq \rho(\varepsilon) .
$$

In case that for $C>0$, we consider $\rho(t)=C t$ for all $t \geq 0$ then the fixed point equation (20) is said to be Ulam type stable.

On a metric space $(x, d)$, the fixed point problem (20), where $f: x \rightarrow \chi$, is said to be well-posed if the following assumptions are satisfy:

1. $f$ has a unique fixed point $z$ in $x$;

2. $d\left(x_{n}, z\right)=0$ for each sequence $\left\{x_{n}\right\} \in X$ such that $\lim _{n \rightarrow \infty} d\left(x_{n}, f x_{n}\right)=0$.

Theorem 3.1. Let $(x, d)$ be a complete metric space. If we add the condition $\lambda_{1}+\lambda_{5}<\frac{1}{c^{2}(q)}$, where $c(q)=$ $\max \left\{1,2^{q-1}\right\}$, to the assumptions of Theorem 2.5, then the following affirmations hold:

(i) the fixed point equation (20) is Ulam-Hyers stable if $\alpha(u, v) \geq 1$ for any $u$, $v$ satisfying the inequality (21);

(ii) the fixed point equation (20) is well-posed if $\alpha\left(x_{n}, z\right) \geq 1$ for any sequence $\left\{x_{n}\right\} \in x$ such that $\lim _{n \rightarrow \infty} d\left(x_{n}, f \chi_{n}\right)=0$ and $\operatorname{Fix}_{f}(x)=z$.

Proof. (i) Since from Theorem 2.5 we know that there is an unique $z \in X$ such that $f z=z$, let $y^{\star} \in X$ such that

$$
d\left(y^{\star}, f_{y}^{\star}\right) \leq \varepsilon, \text { for } \varepsilon>0 .
$$


Obvious, $z$ verifies (21) so we have that $\alpha\left(y^{*}, z\right) \geq 1$ and then by using the triangular inequality we get

$$
\begin{aligned}
& d\left(z, y^{\star}\right) \leq d\left(f z, f y^{*}\right)+d\left(f y^{\star}, y^{\star}\right) \leq \alpha\left(y^{*}, z\right) d\left(f y^{*}, f z\right)+d\left(f y^{\star}, y^{\star}\right) \\
& \leq \psi\left(\mathcal{R}_{f}^{d}\left(y^{*}, z\right)\right)+d\left(f y^{*}, y^{*}\right)<\mathcal{R}_{f}^{d}\left(y^{\star}, z\right)+d\left(f y^{*}, y^{\star}\right) \\
& \leq\left[\lambda_{1} d^{q}\left(z, y^{*}\right)+\lambda_{2} d^{q}\left(y^{*}, f y^{*}\right)+\lambda_{3} d^{q}(z, f z)+\lambda_{4}\left(\frac{d(z, f z)\left(1+d\left(y^{*}, f y^{*}\right)\right)}{1+d\left(y^{*}, z\right)}\right)^{q}\right. \\
& \left.+\lambda_{5}\left(\frac{d\left(z, f y^{*}\right)\left(1+d\left(y^{*}, f z\right)\right)}{1+d\left(y^{*}, z\right)}\right)^{q}\right]^{\frac{1}{q}}+d\left(f y^{*}, y^{*}\right) \\
& =\left[\lambda_{1} d^{q}\left(z, y^{\star}\right)+\lambda_{2} d^{q}\left(y^{\star}, f y^{\star}\right)+\lambda_{3} d^{q}(z, z)+\right. \\
& \left.+\lambda_{4}\left(\frac{d(z, z)\left(1+d\left(y^{*}, f y^{*}\right)\right)}{1+d\left(y^{*}, z\right)}\right)^{q}+\lambda_{5}\left(\frac{d\left(z, f y^{*}\right)\left(1+d\left(y^{*}, z\right)\right)}{1+d\left(y^{*}, z\right)}\right)^{q}\right]^{\frac{1}{q}}+d\left(f y^{*}, y^{*}\right) \\
& \leq\left[\lambda_{1} d^{q}\left(z, y^{*}\right)+\lambda_{2} \varepsilon^{q}+\lambda_{5} d^{q}\left(z, f y^{*}\right)\right]^{\frac{1}{q}}+\varepsilon \\
& \leq\left[\lambda_{1} d^{q}\left(z, y^{\star}\right)+\lambda_{2} \varepsilon^{q}+\lambda_{5}\left(d\left(z, y^{\star}\right)+d\left(y^{\star}, f y^{*}\right)\right)^{q}\right]^{\frac{1}{q}}+\varepsilon \\
& \leq\left[\lambda_{1} d^{q}\left(z, y^{*}\right)+\lambda_{2} \varepsilon^{q}+\lambda_{5}\left(d\left(z, y^{*}\right)+\varepsilon\right)^{q}\right]^{\frac{1}{q}}+\varepsilon \text {. }
\end{aligned}
$$

Therefore,

$$
\begin{aligned}
d^{q}\left(z, y^{\star}\right) & \leq c(q)\left[\lambda_{1} d^{q}\left(z, y^{\star}\right)+\lambda_{2} \varepsilon^{q}+\lambda_{5}\left(d\left(z, y^{\star}\right)+\varepsilon\right)^{q}+\varepsilon^{q}\right] \\
& \leq c(q)\left[\lambda_{1} d^{q}\left(z, y^{\star}\right)+\lambda_{2} \varepsilon^{q}+\lambda_{5} c(q)\left(d^{q}\left(z, y^{\star}\right)+\varepsilon^{q}\right)+\varepsilon^{q}\right],
\end{aligned}
$$

where $c(q)=\max \left\{1,2^{q-1}\right\}$. By simple calculation, from the above inequality we have

$$
d^{q}\left(z, y^{\star}\right) \leq \frac{\left(1+\lambda_{2}+c(q) \lambda_{5}\right) c(q)}{1-c(q) \lambda_{1}-c^{2}(q) \lambda_{5}} \varepsilon^{q},
$$

which is equivalent with

$$
d\left(z, y^{\star}\right) \leq C \varepsilon,
$$

where $C=\left(\frac{\left(1+\lambda_{2}+c(q) \lambda_{5}\right) c(q)}{1-c(q) \lambda_{1}-c^{2}(q) \lambda_{5}}\right)^{\frac{1}{q}}$, for any $q>0$ and $\lambda_{1}, \lambda_{5} \in[0,1)$ such that $\lambda_{1}+\lambda_{5}<\frac{1}{c^{2}(q)}$.

(ii) Taking into account the supplementary condition and since $\operatorname{Fix}_{f}(x)=z$ we have

$$
\begin{aligned}
& d\left(x_{n}, z\right) \leq d\left(x_{n}, f x_{n}\right)+d\left(f x_{n}, f z\right) \\
& \leq d\left(x_{n}, f x_{n}\right)+\alpha\left(x_{n}, z\right) d\left(f x_{n}, f z\right) \\
& \leq d\left(x_{n}, f x_{n}\right)+\psi\left(\mathcal{R}_{f}^{d}\left(x_{n}, z\right)\right) \\
& <d\left(x_{n}, f x_{n}\right)+\mathcal{R}_{f}^{d}\left(x_{n}, z\right) \\
& \leq\left[\lambda_{1} d^{q}\left(x_{n}, z\right)+\lambda_{2} d^{q}\left(x_{n}, f x_{n}\right)+\lambda_{3} d^{q}(z, f z)\right. \\
& \left.+\lambda_{4}\left(\frac{d(z, f z)\left(1+d\left(x_{n}, f x_{n}\right)\right)}{1+d\left(x_{n}, z\right)}\right)^{q}+\lambda_{5}\left(\frac{d\left(z, f_{n}\right)\left(1+d\left(x_{n}, f z\right)\right)}{1+d\left(x_{n}, z\right)}\right)^{q}\right]^{\frac{1}{q}}+d\left(x_{n}, f x_{n}\right) \\
& =\left[\lambda_{1} d^{q}\left(x_{n}, z\right)+\lambda_{2} d^{q}\left(x_{n}, f x_{n}\right)+\lambda_{5} d^{q}\left(z, f x_{n}\right)\right]^{\frac{1}{q}}+d\left(x_{n}, f x_{n}\right) \\
& \leq\left[\lambda_{1} d^{q}\left(\chi_{n}, z\right)+\lambda_{2} d^{q}\left(\chi_{n}, f \chi_{n}\right)+\lambda_{5}\left(d\left(z, \chi_{n}\right)++d\left(\chi_{n}, f \chi_{n}\right)\right)^{q}\right]^{\frac{1}{q}}+d\left(\chi_{n}, f \chi_{n}\right) \\
& \leq\left[\lambda_{1} d^{q}\left(x_{n}, z\right)+\lambda_{2} d^{q}\left(x_{n}, f x_{n}\right)+\lambda_{5} c(q)\left(d^{q}\left(z, x_{n}\right)+d^{q}\left(x_{n}, f x_{n}\right)\right)\right]^{\frac{1}{q}}+d\left(x_{n}, f x_{n}\right),
\end{aligned}
$$

or,

$$
d\left(x_{n}, z\right)^{q} \leq \frac{\left(1+\lambda_{2}+c(q) \lambda_{5}\right) c(q)}{1-c(q) \lambda_{1}-c^{2}(q) \lambda_{5}} d^{q}\left(x_{n}, f x_{n}\right) .
$$

Letting $n \rightarrow \infty$ in the above inequality and keeping in mind that $\lim _{n \rightarrow \infty} d\left(\chi_{n}, f \chi_{n}\right)=0$, we obtain

$$
\lim _{n \rightarrow \infty} d\left(x_{n}, z\right)=0
$$

that is, the fixed point equation (20) is well-posed. 


\section{References}

[1] Rus I. A., Generalized Contractions and Applications, Cluj University Press: Clui-Napoca, Romania, 2001

[2] Bianchini R. M., Grandolfi M., Transformazioni di tipo contracttivo generalizzato in uno spazio metrico, Atti Acad. Naz. Lincei, VII. Ser. Rend. Cl. Sci. Fis. Mat. Natur., 1968, 45, 212-216

[3] Popescu 0., Some new fixed point theorems for $\alpha$-Geraghty contractive type maps in metric spaces, Fixed Point Theory Appl., 2014, 2014:190

[4] Agarwal R. P., Karapinar E., Interpolative Rus-Reich-Ćirić type contractions via simulation functions, An. St. Univ. Ovidius Constanta, Ser. Mat., (in press)

[5] Aydi H., Chen C. M., Karapinar E., Interpolative Ćirić-Reich-Rus type contractions via the Branciari distance, Mathematics, 2019, 7(1), 84

[6] Aydi H., Karapinar E., Roldan Lopez de Hierro A. F., $\omega$-interpolative Ćirić-Reich-Rus-type contractions, Mathematics, 2019, 7(1), 57

[7] Karapinar E., Alqahtani O., Aydi H., On interpolative Hardy-Rogers type contractions, Symmetry, 2019, 11(1), 8

[8] Karapinar E., Revisiting the Kannan type contractions via interpolation, Adv. Theory Nonlinear Anal. Appl., 2018, 2, 85-87

[9] Karapinar E., Agarwal R., Aydi H., Interpolative Reich-Rus-Ćirić type contractions on partial metric spaces, Mathematics, 2018, 6, 256

[10] Ulam S. M., Problems in Modern Mathematics, John Wiley and Sons, New York, NY, USA, 1940

[11] Ulam S. M., A Collection of Mathematical Problems, Interscience, New York, NY, USA, 1968

[12] Hyers D. H., On the stability of linear functional equations, Proc. Natl. Acad. Sci., USA, 1941, 27, 222-224

[13] Rassias T. M., On the stability of linear mapping in Banach Spaces, Proc. Am. Math. Soc., 1978, 72, 297-300 